\title{
Spatial distribution and variation of risk factors for poor consumption of foods rich in vitamin A among children age 6-23 months in Ethiopia. Geographical weighted regression analysis
}

Sofonayas Abebaw Tiruneh ( $D$ zephab2@gmail.com )

Debre Tabor University https://orcid.org/0000-0001-5867-5105

Dawit Tefera Fentie

University of Gondar

Seblewongel Tigabu Yigizaw

University of Gondar

Asnakew Asmamaw Abebe

University of Gondar

Kassahun Alemu Gelaye

University of Gondar

Research article

Keywords: Vitamin A, Spatial distribution, GWR, Children, Ethiopia

Posted Date: October 30th, 2019

DOl: https://doi.org/10.21203/rs.2.16631/v1

License: (c) (1) This work is licensed under a Creative Commons Attribution 4.0 International License.

Read Full License 


\section{Abstract}

Introduction: Vitamin A deficiency is a major nutritional public health problem in poor societies. Dietary consumption of foods rich in vitamin A was low in Ethiopia. This study aimed to assess the spatial distribution and its determinants of dietary consumption of foods rich in vitamin A among children age 623 months in Ethiopia.

Methods: A total of 3055 children were included and data were accessed from 2016 Ethiopian Demographic and Health Survey dataset . The data was cleaned and weighted by STATA version 14.1 software and Microsoft excel. The Bernoulli model fitted using Kuldorff's SaTScan version 9.6 software. ArcGIS version 10.7 software was used to visualize spatial distribution for poor consumption of foods rich in vitamin A. Geographical weighted regression analysis was employed by MGWR version 2.0 software. A P-value of less than 0.05 was used to declare statistically significant predictors locally.

Results: Overall, $62 \%(95 \% \mathrm{Cl}: 60.56,64.00)$ of children age $6-23$ months had poor consumption of foods rich in Vitamin A in Ethiopia. Poor consumption of foods rich in vitamin A highly clustered at Afar, eastern Tigray, southeast of Amhara, and eastern Somali region of Ethiopia. Spatial scan statistics identified 142 primary spatial clusters located at Afar, the eastern part of Tigray, most part of Amhara and some part of Oromia Regional State of Ethiopia. Children living in the primary cluster were $46 \%$ more likely venerable poor consumption of foods rich in vitamin $A$ than outside the window $(R R=1.46, L L R=83.78, P<0.001)$. Poor wealth status of the household, rural residence and living tropical area of Ethiopia were statistically significant predictors spatially.

Conclusion: Overall, the consumption of foods rich in vitamin A was low and spatially non-random in Ethiopia. Poor wealth status of the household, rural residence and living tropical area were significant predictors for the consumption of foods rich vitamin A locally in Ethiopia. Policymakers and health planners should intervene in nutrition intervention at the identified hot spot areas to reduce poor consumption of foods rich in vitamin A among children age 6-23 months.

\section{Introduction}

Vitamin A is a fat-soluble vitamin used for formation rhodopsin, photoreceptor pigment of the retina, helps maintain epithelial tissues, and immune enhancer (1). Mainly performed retinol and provitamin A carotenoids foods rich in vitamin A were available. Preformed retinol foods rich in vitamin A were exclusively found in animal products and provitamin A carotenoids found in green leafy vegetables (2).

Low consumption of foods rich in vitamin A during nutritionally demanding periods in life, such as infancy and childhood consequences vitamin A deficiency disorders (3). Its deficiency was associated with measles, Diarrhoea, malaria and other infectious disease morbidity and mortality among children $(4,5)$. 
Vitamin A deficiency (VAD) is a major nutritional public health problem in poor societies, especially in lowincome countries. The magnitude of vitamin A deficiency (VAD) was highest in Sub-Saharan Africa (48\%; 25-75) and South Asia (44\%; 13-79). In 2013, 94500 (54 200-146 800) deaths from diarrhoea and 11 200 (4300-20 500) deaths from measles were attributable to vitamin A deficiency in Sub-Saharan Africa and South Asia among children (4). The estimated prevalence of vitamin A deficiency among children in Ethiopia ranges between $20-39 \%(1,4)$.

Dietary consumption of foods rich in vitamin A affected by socio-economic and demographic status of the household (6,7), maternal knowledge and media exposure about Infant and Young Child Feeding (IYCF) and husband involvement in IYCF (8), maternal and husband education $(7,9)$, and media exposure $(9,10)$ were some of the factors significantly affects dietary consumption.

Vitamin A supplementation is associated with a clinically meaningful reduction in morbidity and mortality in children (5). A meta-analysis of 17 trials evidenced that vitamin consumption reduces allcause mortality the overall risk of death by $24 \%$ among children. Another trial evidenced that consumption of foods rich in vitamin A and supplementation significantly reduces diarrhoea-related mortality by $28 \%$ among children (11).

Despite the importance of vitamin A for children, the consumption of foods rich in vitamin A in Ethiopia remains low. Previous studies evidenced that only $7 \%$ to $39 \%$ of children age 6-23 months consumed plant source foods rich in vitamin A in Ethiopia $(6,8,12)$. On the other hand, only 12 to $24 \%$ of children age 6-23 months consumed animal source foods rich in vitamin A in Ethiopia $(6,12)$, however, eggs $(11.0 \%)$ and meat $(2.6 \%)$ were less frequently consumed (8).

So far, different studies conducted in Ethiopia to assess dietary diversity among children including foods rich in vitamin A consumption. But there is no evidence conducted to know the spatial distribution of dietary consumption of foods rich vitamin A across the regions of Ethiopia. Knowing the spatial distribution of dietary consumption of foods rich in vitamin $A$ in the regions of Ethiopia used for local specific nutrition intervention to tackle vitamin A deficiency-related child morbidity and mortality.

Therefore, the objective of this study was to assess the spatial distribution of dietary consumption of foods rich in vitamin A and its spatial determinants among children age 6-23 months in Ethiopia.

\section{Methods And Materials}

Study design, area and period:

This study is a community-based cross-sectional study was conducted using nationally representative 2016, Ethiopian Demographic and Health Survey (EDHS) dataset. Ethiopia is situated in the Horn of Africa from $3^{0}$ to $14^{0}$ and $33^{0}$ to $48^{\circ} \mathrm{E}$.

Source and study populations: 
The source population was all living children age 6-23 months living with their motherpreceding the survey. The study population was all living children age 6-23 months living with their mother and the mother was interviewed preceding the survey in the selected Enumeration Areas (EAs). In 2016 EDHS, a total of 645 clusters (EAs) (202 urban and 443 rural) were selected with a probability proportional to each EAs size and independent selection in each sampling stratum. Among the total of selected clusters with zero coordinates and clusters without a proportion of children status of consumption of foods rich in vitamin A was excluded for the analysis. Finally, a total of 598 (185 urban and 413 rural) cluster were used for this study. Among the selected clusters a total of 3055 weighted living children age 6-23 months living with their mother and interviewed for this study. The recorded data were accessed at www.measuredhs.com on request with the help of ICF International, Inc.

\section{Data collection tools and procedures:}

Ethiopian Demographic and Health Survey data were collected by two-stage stratified sampling. Each region of the country was stratified into urban and rural areas, yielding 21 sampling strata. In the first stage, 645 EAs were selected with probability proportional to Enumeration Area size by independent selection in each sampling stratum. In the second stage of selection, a fixed number of 28 households per cluster were selected with an equal probability systematic sampling from the newly created household listing. The detail sampling procedure was available in the Ethiopian Demographic and Health Survey reports from Measure DHS website (www.dhsprogram.com).

\section{Outcome variable:}

Living children age 6-23 months living with their mother who consumed foods rich in vitamin A at least one food item among the seven food items at any time in the last 24 hours preceding the interview was declared good consumption of foods rich in vitamin $A$, whereas, none consumption of foods rich in vitamin $A$ in 24 hours preceding the interview was poor consumption.

\section{Predictor variables:}

From the 2016 EDHS datasets, the proportion of mothers age, educational status of mother and husband, residence, religion of mother, parity, wealth status, and altitude were considered predictor variables.

\section{Data management and analysis:}

The data was cleaned by STATA version 14.1 software and Microsoft excel. Sample weighting was done for further analysis.

\section{Spatial autocorrelation and hot spot analysis:}

Spatial autocorrelation (Global Moran's I) statistic measure was used to assess whether the consumption of foods rich in vitamin A among children age 6-23 months was dispersed, clustered, or randomly distributed in Ethiopia. Moran's I values close to -1 indicates poor consumption of foods rich in vitamin A 
dispersed, close to +1 indicates clustered, and if Moran's I value zero indicates randomly distributed (13). A statistically significant Moran's I value $(p<0.05)$ had a chance to rejection of the null hypothesis which indicates the presence of spatial autocorrelation. Hot Spot Analysis (the Getis-Ord Gi* statistic) of the zscores and significant $p$-values tells the features with either hot spot or cold spot values for the clusters spatially.

\section{Spatial interpolation:}

Spatial interpolation technique is used to predict poor consumption of foods rich in vitamin A among children age 6-23 months for unsampled areas in the country based on sampled EAs. For the prediction of unsampled EAs, we used deterministic and geostatistical Empirical Bayesian Kriging spatial interpolation technique. Empirical Bayesian Kriging relaxes the assumption of Gaussian distribution of the observed semivariogram in the input data which rarely holds true in practice. Empirical Bayesian Kriging interpolation works by generating a new simulated semivariogram at each location from the estimated semivariogram from the input data. Weight of the new simulated semivariogram is calculated by Bayes' rule (14).

\section{Spatial scan statistics:}

We employed Bernoulli based model spatial scan statistics to determine the geographical locations of statistically significant clusters for poor consumption of foods rich in vitamin A among children age 623 months using Kuldorff's SaTScan version 9.6 software (15). The scanning window that moves across the study area which children age 6-23 months with poor consumption of foods rich in vitamin A were taken as cases and those good consumption taken as controls to fit the Bernoulli model. The default maximum spatial cluster size of $<50 \%$ of the population was used as an upper limit, allowing both small and large clusters to be detected, and ignored clusters that contained more than the maximum limit with the circular shape of the window. Most likely clusters were identified using p-values and likelihood ratio tests on the basis of the 999 Monte Carlo replications.

\section{Geographically weighted regression analysis:}

Ordinary Least Square regression (OLS) model is a global model, which estimates only one single coefficient per explanatory variable over the entire study area. Global models assume factors that affect poor consumption of foods rich in vitamin A were stationary geographically. The assumption of geographical independence may bias the parameter estimates. The assumption of geographical independence relaxes by geographically weighted regression analysis. A geographically weighted regression model is an extension of OLS regression model and gives local parameter estimates to reflect changes over space in the association between an outcome and explanatory variables (13).

For the interest of geographically weighted regression analysis, the aggregated proportion of poor consumption of foods rich in vitamin A among children age 6-23 months and all the predictor variable were calculated for each cluster. To determine the predictor variables for poor consumption of foods rich 
in vitamin A among children age 6-23 months, we used Geographically Weighted Regression (GWR) model.

To check the assumption of spatial dependency explanatory analysis was performed first by Arc GIS 10.7 software. Statistically significant $(P<0.01)$ Koenker $(B P)$ statistic indicates that the relationships modelled are not consistent (either due to non-stationarity or heteroskedasticity). Multicollinearity (Variance Inflation Factor $<7.5$ ) were checked to exclude redundancy among explanatory variables. In the case of spatial dependency, the coefficient of predictor variable varies locally as well the predictor variables may or may not significant locally. The model structure of geographically weighted regression written as,

\section{$Y_{i}=\beta_{0}\left(u_{i}, v_{i}\right)+\sum_{k} \beta_{k}\left(u_{i}, v_{i}\right) X_{i k}+\square_{i}$}

Where $Y_{i}$ is the response variable, $\left(u_{i}, v_{i}\right)$ denotes the coordinates of the $i^{i t h}$ point in space, $\beta_{0}$ is the intercept atthe $\left(u_{i}, v_{i}\right)$ coordinate, $\beta_{k}$ is the coefficient of the covariate $X$ at the $\left(u_{i}, v_{i}\right)$ coordinate, and $\nabla_{i}$ is the random error term.

\section{Calibration of the model:}

We used Multiscale Geographically Weighted Regression (MGWR) version 2.0 software to calibrate the parameter estimates of the Geographically Weighted Regression (GWR) model (16). The new version of GWR is termed Multiscale Geographically Weighted Regression (MGWR), was potentially providing a more flexible and scalable framework in which to examine multiscale processes. Adaptive bi-square kernels were used for geographical weighting to estimate local parameter estimates. The golden section search method was used to determine the best bandwidth size based on corrected Akaike's Information Criterion (AICC) and the bandwidth with the lowest AICc was used to determine the best fit model for local parameter estimates.

Geographical variability for each coefficient can be assessed by comparing the AICc between the GWR model and the global OLS regression model. The corrected Akaike's Information Criterion (AICc) was obtained by minimizing the Akaike Information Criteria (AIC) which is;

[See supplementary file for formula]

where $\mathrm{n}$ is the sample size, $\sigma^{\wedge}$ is the estimated standard deviation of the error term, and $\operatorname{tr}(S)$ denotes the trace of the hat matrix which is a function of the bandwidth. Finally, local parameter estimates were plotted on Arc GIS 10.7(ESRI Inc., Redlands, CA, USA, version 10.7) software.

\section{Ethical consideration:}

We, authors, submitted a proposal to DHS Program/ICF International Inc, and permission was confirmed from the International Review Board of Demographic and Health Surveys (DHS) program data archivist to 


\section{Results}

characteristics of the respondents and study children:

A total of 3055 children age 6-23 months included for this study. More than half (53\%) of children were females. From the total of children, $18.45 \%$ between age $6-8$ months and $36.6 \%$ were age 12-17 months. The mean \pm SD age of the children was $13.92 \pm 5.05$ months. The majority (67.50\%) of the mothers were in the age group of 20-34 years. The mean \pm SD age of the mothers was $28.25 \pm 6.47$ years. All most $(94 \%)$ of mothers were married. About $61 \%$ of mothers and $45 \%$ of husbands had no formal education. Approximately $59 \%$ of mothers and $11 \%$ of husbands had no work during the survey period. Regarding residence, $88 \%$ of children live in a rural area and $45 \%$ of them were living in a tropical region. Forty-four per cent of the household was poor wealth status. (Table 1)

Table 1: Sociodemographic characteristics of the respondents and study children age 6-23 months in Ethiopia, EDHS 2016. $(n=3055)$ 


\begin{tabular}{|c|c|c|c|}
\hline \multicolumn{2}{|c|}{ Variables } & Frequency (n) & Per cent (\%) \\
\hline \multirow[t]{3}{*}{ Mother's age (years) } & $<20$ & 369 & 12.08 \\
\hline & $20-34$ & 2,062 & 67.50 \\
\hline & $35-49$ & 624 & 20.42 \\
\hline \multirow[t]{2}{*}{ Marital status } & Married & 2864 & 93.76 \\
\hline & Not married & 191 & 6.24 \\
\hline \multirow[t]{3}{*}{ Religion } & Orthodox & 1,049 & 34.35 \\
\hline & Muslim & 1,236 & 40.45 \\
\hline & Others & 770 & 25.20 \\
\hline \multirow[t]{3}{*}{ Mother's education } & No education & 1,864 & 61.03 \\
\hline & Primary education & 936 & 30.62 \\
\hline & Secondary and above & 255 & 8.35 \\
\hline \multirow[t]{3}{*}{ Husband's education } & No education & 1,291 & 44.55 \\
\hline & Primary education & 1,198 & 41.34 \\
\hline & Secondary and above & 408 & 14.10 \\
\hline \multirow[t]{2}{*}{ Mother occupation } & Working & 1,265 & 41.41 \\
\hline & Not working & 1,790 & 58.59 \\
\hline \multirow[t]{2}{*}{ Husband occupation } & Working & 2,717 & 88.93 \\
\hline & Not working & 338 & 11.07 \\
\hline \multirow[t]{2}{*}{ Family size } & Less than three & 1,650 & 54.00 \\
\hline & Greater than four & 1,405 & 46.00 \\
\hline \multirow[t]{4}{*}{ Child age (months) } & $6-8$ months & 564 & 18.45 \\
\hline & $9-11$ months & 502 & 16.43 \\
\hline & 12 - 17 months & 1,118 & 36.59 \\
\hline & 18 - 23 months & 871 & 28.53 \\
\hline \multirow[t]{2}{*}{ Child sex } & Male & 1,433 & 46.90 \\
\hline & Female & 1,622 & 53.10 \\
\hline \multirow[t]{2}{*}{ Media exposure } & No media exposure & 2,045 & 66.92 \\
\hline & Had media exposure & 1,010 & 33.08 \\
\hline \multirow[t]{3}{*}{ Household wealth } & Poor & 1,350 & 44.18 \\
\hline & Middle & 683 & 22.37 \\
\hline & Rich & 1,022 & 33.44 \\
\hline \multirow[t]{2}{*}{ Residence } & Rural & 2,684 & 87.85 \\
\hline & Urban & 371 & 12.15 \\
\hline \multirow[t]{4}{*}{ Altitude } & Tropical & 1,362 & 44.58 \\
\hline & Sub-tropical & 1,159 & 37.92 \\
\hline & Dega & 534 & 17.50 \\
\hline & Total & 3055 & 100 \\
\hline
\end{tabular}


Consumption status of Foods rich in Vitamin A among children age 6-23 months. Overall, two-third (62\%: 95\% CI: 60.56, 64.00) of children age 6-23 months had poor consumption of foods rich in Vitamin A. Animal source foods rich in vitamin A were reported primarily poor in consumption preceding the last 24 hours in the survey period. Egg consumption was reported mostly taken foods in the last 24 hours period. (Table 2)

Table 2: Consumption of foods rich in Vitamin A among children age 6-23 months in the last 24-hours preceding survey 2016, EDHS, Ethiopia. $(\mathrm{n}=3055)$

Food groups interviewed in the last 24 hours.

Consumption status

. Good Poor

(\%)

Have the child took eggs in the last 24 hours?

$16.81 \quad 83.19$

Have the child took meat (beef, pork, lamb, chicken, etc.) in the last $\quad 5.95 \quad 94.05$ 24 hours?

Have the child took a pumpkin, carrots, squash (yellow or orange inside) in the last 24 hours?

$12.17 \quad 87.83$

Have child took any dark green leafy vegetables in the last 24 hours?

$13.56 \quad 86.44$

Have the child took mangoes, papayas, other vitamin A fruits in the last 24 hours?

$12.83 \quad 87.17$

Have the child took liver, heart, other organs in the last 24 hours?

$3.89 \quad 96.11$

Have the child took fish or shellfish in the last 24 hours?

$1.31 \quad 98.69$

erall consumption of foods rich in vitamin A among children age 6-23 $\quad 32.70 \quad 62.30$ inths.

Spatial distribution poor consumption of foods rich in vitamin A among children age 6-23 months.

To determine spatial clustering for poor consumption of foods rich in vitamin A, global spatial statistics was estimated using Moran's I value. As shown the figure below a statistically significant z-scores indicate at $152 \mathrm{Km}$ distances where spatial processes promoting clustering are most pronounced. The incremental spatial Autocorrelation 
indicates that totally 8 distance bands were detected with a beginning distance of 120000 meters. The spatial distribution of poor consumption of foods rich in vitamin A among children age 6-23 months in Ethiopia was found non-random with a Global Maran's I was 0.21 and p-value 0.001 . The z-score of 14.13 , there is a less than $1 \%$ likelihood that this high-clustered pattern could be the result of random chance. (Fig 1)

\section{Hot spot (Getis-Ord Gi*) analysis:}

As shown in the figure below, the red color indicates the more intense clustering of high (hot spot) proportion with poor consumption of foods rich in vitamin A preceding the survey period. A high proportion of poor consumption of foods rich in vitamin A among children age 6-23 months were clustered at Afar, eastern Tigray, southeast of Amhara, and eastern Somali region of Ethiopia. Whereas, Addis Ababa, Gamebela, and Central Oromia regions of Ethiopia were less risk area for poor consumption of foods rich in vitamin A among children age 6-23 months. (Fig 2)

\section{Spatial scan statistics analysis:}

In spatial scan analysis, a total of 187 significant clusters were identified. As shown in figure 3 below, the red window indicates the significant clusters. Among the significant clusters, 142 were most likely (primary) and 45 were secondary clusters. The most likely (primary) clusters were located at $11.626646 \mathrm{~N}, 39.666950 \mathrm{E}$ in $278.08 \mathrm{~km}$ radius in Afar, the eastern part of Tigray, and most part of Amhara National Regional State of Ethiopia. The secondary significant clusters were located at $6.745502 \mathrm{~N}, 44.259010 \mathrm{E}$ in $360.64 \mathrm{~km}$ radius in Somali and some part of Oromia National Regional State of Ethiopia. Children age 6-23 months living in the primary cluster were $46 \%$ more likely vulnerable to poor consumption of foods rich in vitamin A than outside the window ( $R R=1.46$, $L L R=83.78$, P-value < 0.001). Children living in the secondary cluster were $36 \%$ more likely to risk of poor consumption of foods rich in vitamin A as compared to outside the window $(R R=1.36$, LLR $=27.18$, P-value $<0.001$ ). (Table 3 \& Fig 3)

Table 3: Significant spatial scan clusters of poor consumption of foods rich in vitamin A among children age 6-23 months, EDHS, 2016. 


\begin{tabular}{|c|c|c|c|c|c|c|c|}
\hline r type & $\begin{array}{l}\text { Significant } \\
\text { Enumeration } \\
\text { Areas(clusters) } \\
\text { detected }\end{array}$ & $\begin{array}{l}\text { Coordinates } \\
\text { /Radius }\end{array}$ & Populations & Cases & RR & LLR & $\begin{array}{l}\mathrm{P}- \\
\text { value }\end{array}$ \\
\hline
\end{tabular}




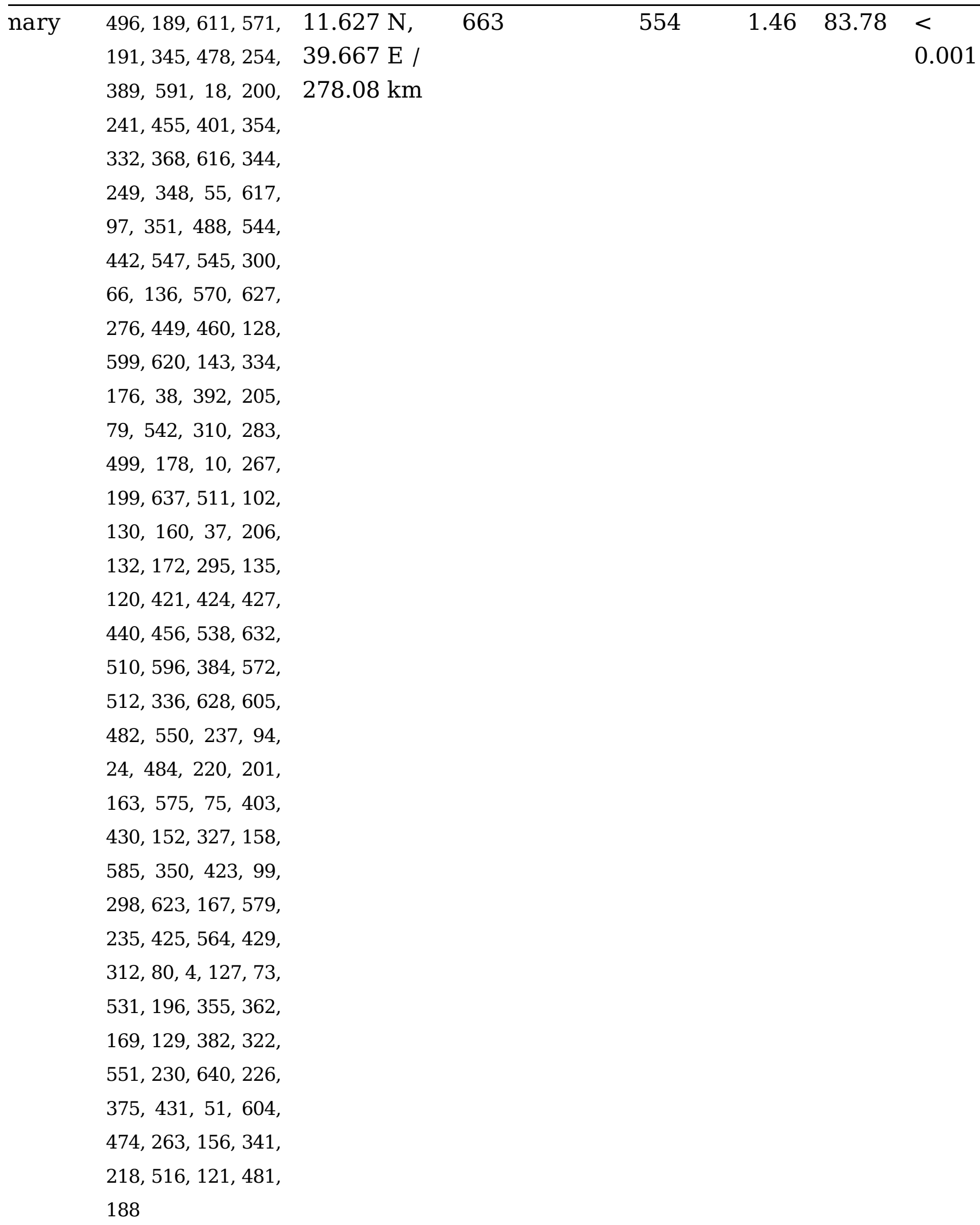




\begin{tabular}{|c|c|c|c|c|c|c|c|}
\hline ndary & $\begin{array}{l}490,543,92,492, \\
171,198,146,95, \\
85,358,164,138, \\
497,521,588,458, \\
553,278,77,629, \\
214,318,251,573, \\
187,239,116,22 \\
33,568,277,527, \\
\begin{array}{l}269,556,630,64, \\
439,57,480,8, \\
210,186,454,436, \\
566\end{array}\end{array}$ & $\begin{array}{l}6.745 \mathrm{~N}, \\
44.259 \mathrm{E} / \\
360.64 \mathrm{~km}\end{array}$ & 250 & 209 & 1.36 & 27.18 & $\begin{array}{l}< \\
0.001\end{array}$ \\
\hline
\end{tabular}

NB: $R R=$ relative risk, LLR $=$ Log-Likelihood Ratio.

Prevalence of poor consumption of foods rich in vitamin A among children age 6-23 months.

Most parts of the regions in Ethiopia, children age 6-23 months were venerable to poor consumption of foods rich in vitamin A. Children in Afar, eastern Amhara, and Eastern Somalia regions of Ethiopia were more venerable to poor consumption of foods rich in vitamin A as compared to other regions of Ethiopia. (Fig 4)

\section{Geographically weighted regression Analysis:}

Selected predictor variables fitted in the geographically weighted regression model. For model compression, both Ordinary Least Square (OLS) model with Geographical Weighted Regression (GWR) model was fitted. The bandwidth corrected Akakian Information Criteria (AICC), adjusted $\mathrm{R}^{2}$, and loglikelihood were considered for model comparison. Comparing the global model, Geographical Weighted Regression was the best fit model with AICc of 1570 compared with 1616. As well, the GWR model best explained by the predictor variables for poor consumption of foods rich in vitamin A among children age 6-23 months with an adjusted $\mathrm{R}^{2}$ value $62 \%$ compared to $28 \%$. (Table 4 ).

Table 4: Model comparison between the OLS model with GWR model.

\begin{tabular}{lll}
\hline Values & OLS Model & GWR Model \\
\hline AICc & 1616.27 & 1570.65 \\
\hline Adjusted R & $28 \%$ & $62 \%$ \\
\hline Log likelihood & -788.48 & -742.23 \\
\hline
\end{tabular}


NB: AICc $=$ corrected Akakian Information Criteria

In Geographically weighted Regression model predictor variables poor wealth status of the household, rural residence, and living in the tropical area were statistically significant spatially for poor consumption of foods rich in vitamin A among children age 6 to 23 months. The strength of the association with predictor variables varies spatially and the effects of variables had a positive and negative effect locally.

Poor wealth status of the household had different statistical significance in different parts of Ethiopia for poor consumption of foods rich in vitamin A among children age 6 to 23 months. The coefficients of poor wealth status vary spatially between 0.088 to 0.203 indicating that the effect of association different across regions of Ethiopia. In the significant parts of Ethiopia, a $1 \%$ increase in poor wealth status proportion of the household will increase the prevalence of poor consumption of foods rich in vitamin A among children age 6 to 23 months between $14.6 \%$ to $20.3 \%$. Poor wealth status was not statistically significant in Benishangul Regional state, most part of Tigray and Amhara Regional state, and some part of Oromia and Gambela regional state of Ethiopia (Fig 5).

Residence was statistically significant for poor consumption of foods rich in vitamin A over regions of Ethiopia. The coefficients of rural residence vary spatially from -0.072 to 0.309 , which indicates rural residence were had a negative and positive effect spatially for poor consumption of foods rich in vitamin A among children age 6-23 months. Keeping constant other factors, living rural area had increased the risk of poor consumption foods rich in vitamin A by $22.5 \%$ to $31 \%$. (Fig 6 )

Furthermore, children living in the tropical areas of Ethiopia were had different spatial significance fo poor consumption of foods rich in vitamin A. The effects of living tropical area on poor consumption of foods rich in vitamin A among children age 6-23 months vary between -0.284 to 0.235 that indicates both negative and positive effect on vitamin A rich foods consumption. Being constant other factors, children living in tropical area of Benishangul, western part of Amhara and Oromia, and Gambela regions of Ethiopia were decrease the risk of poor consumption foods rich in vitamin A by a factor of between $19 \%$ to $29 \%$, whereas children live in tropical area of Somalia regional state of Ethiopia had increase poor consumption of foods rich in vitamin A. (Fig 7)

\section{Discussion}

This study revealed that $62.30 \%$ (95\% Cl: $60.56 \%, 64.00 \%)$ of children age $6-23$ months were having poor consumption of foods rich in vitamin A in Ethiopia. The finding of this study lower than a study done Southern Ethiopia (71\%), 2011 Ethiopia Demographic Health survey report $(74 \%)(6,12)$. This finding was higher than the study done Gorche district Ethiopia, 2014 Demographic Health survey report of Kenya (28\%) and Ghana (33\%), and 2016 Demographic Health survey report of India (56\%) (8,17-19). 
Our study showed that the spatial distribution of poor consumption of foods rich vitamin A was nonrandom in Ethiopia. Poor consumption of foods rich in vitamin A highly clustered at Afar, eastern Tigray, southeast Amhara, and the eastern Somali Regional State of Ethiopia. In line with this high proportion of clustering, spatial scan statistics analysis revealed that 187 significant clusters were identified. A high proportion of poor consumption of foods rich in vitamin A in Tigray and Amhara Regional state was supported by Ethiopia national food consumption survey report (20). The observed geographical variation of poor consumption of foods rich in vitamin A across regions of Ethiopia might be due to the regional variation dietary preference, low practice to complementary feeding, socioeconomic status, demographic factor such as pastoralist region, and seasonal differences for the consumption of fruits and vegetables (21).

The local parameter estimates of the predictor variables of the model fit vary spatially in Ethiopia. Poor wealth status of the household, rural residence, and living in the tropical area of Ethiopia were local statistically significant predictor variables for poor consumption of foods rich in vitamin A among children age 6-23 months.

Our study revealed that poor wealth status of the household was locally statistically significant predictor variables for poor consumption of foods rich in vitamin A. In the significant parts of Ethiopia, a $1 \%$ increase proportion of poor wealth status of the household could increase the prevalence of poor consumption of foods rich in vitamin A among children age 6 - 23 months between 14.6\% to 20.3\% (Fig 5 ). The finding of this study supported by the previous study done in Ethiopia, Madagascar children born from the richest households were got adequate dietary diversity and male frequency and Nepal which is Children from the poorest quintile had higher odds of not consuming legumes and nuts, dairy products, flesh foods, and other fruits and vegetables, and not meeting the minimum dietary diversity $(7,10,22,23)$. This finding is not similar to a study done in Ethiopia which is wealth states is not statistically significant to the consumption of foods rich in vitamin A (6). The possible reason might be household with poor wealth did not get minimum meal frequency to their child and poor wealth will affect adherent to the consumption of foods rich in vitamin A and dietary diversity to their child.

Another factor locally affects consumption foods rich in vitamin A was residence. Living rural area had increased the risk of poor consumption foods rich in vitamin $A$ by between $22.5 \%$ to $31 \%$ (Fig. 6 ). This study consistency with the study done in Ethiopia, which is children live in rural area was poor consumption of dietary diversity (24). The possible justification might be living in rural area had no access to get foods rich in vitamin $A$, poor knowledge about foods rich in vitamin $A$, and other socioeconomic factors.

Furthermore, this study revealed that children living in tropical area of Ethiopia were spatially significant to poor consumption of foods rich in vitamin A. Children living in tropical area of Benishangul, western part of Amhara, Oromia, and Gambela region of Ethiopia were decrease the risk of poor consumption foods rich in vitamin A by $19 \%$ to $29 \%$, whereas children living in tropical area of Somalia regional state of Ethiopia had increase poor consumption. The possible discrepancy might be the difference 
accessibility of fruit and vegetable vitamin A rich foods, different cultural practice in the feeding of the child in these different regions of Ethiopia.

Strength and limitation of the study:

As Tobler's first law of geography states that "Everything is related to everything else, but near things are more related than distant things"(25). Based on Tobler's first law of geography, poor consumption of foods rich in vitamin A was spatially autocorrelated. In the presence of spatial dependence and heterogeneity, the estimates obtained from the global model would be biased. Therefore, fitting GWR model and knowing the spatial distribution of poor consumptions of foods rich in vitamin A in regions of Ethiopia provides important insight to policymakers and health planners and valuable hot spot maps used to more effective and cost-efficient nutrition intervention.

The limitation of this study was the coordinates collected at the cluster level not individual-level difficult to do at the individual level and clusters without coordinates were delated may not representative.

\section{Conclusion And Recommendation}

In Ethiopia, poor consumption of foods rich in vitamin A had to vary geographically across regions. Statistically significant-high hot spot of poor consumption of foods rich in vitamin A was identified at Afar, eastern Tigray, southeast of Amhara, and eastern Somali region of Ethiopia, whereas Addis Ababa, Gamebela, and Central Oromia regions of Ethiopia were less risk area. This study showed that predictor variables for poor consumption of foods rich in vitamin A vary spatially in Ethiopia. Poor wealth status of the household, rural residence and living tropical area were statistically significant predictors locally in different regions of Ethiopia. Therefore, policymakers and health planners should design nutrition intervention program at the identified hot spot areas to reduce poor consumption of foods rich in vitamin A among children.

The implication for policymakers and researchers.

The results of this study give rich understanding of the spatial distribution consumption of foods rich in vitamin A among children age 6-23 months in Ethiopia. the expectation of the finding of hos spot maps in line with scan statistics across Ethiopia used to policymakers to give direct nutrition intervention locally. This study focuses on typical consumption of foods rich in vitamin A. the association between dependent and predictor variable might vary across space. In such situation fitting global model may bias the parameter estimates. With this concept, other researchers should be applied GWR model to assess other nutritional problems and dietary diversity among children and reproductive age group mothers, particularly micronutrient insecure areas.

\section{Declarations}

\section{Data Availability}


The data was available from the corresponding author and we can provide upon request.

\section{Consent for publication}

Not applicable

\section{Competing Interests}

We, authors, declare that we had no competing interests.

\section{Funding Statement}

We didn't receive any fund for this study.

\section{Authors' Contributions}

SAT, DTF and STY, were involved for this study from the inception to design, acquisition of data, data cleaning, data analysis and interpretation and drafting and revising of the manuscript. AAA had involved the acquisition of data and data cleaning for the manuscript. KAG was involved in project administration, principal supervision, and revising the final manuscript. All authors read and approved the final manuscript.

\section{Acknowledgements}

We, authors, acknowledge The Demographic and Health Surveys (DHS) Program funded by the U.S. Agency for International Development (USAID) for the accusation dataset.

\section{References}

1. Demissie T, Ali A, Mekonen Y, Haider J, Umeta M. Magnitude and distribution of vitamin A deficiency in Ethiopia. Food Nutr Bull. 2010;31(2):234-41.

2. FAO, WHO. Vitamin and mineral requirements in human nutrition. Second edition. World Heal Organ Food Agric Organ United Nations [Internet]. 2004;1-362. Available from:

https://apps.who.int/iris/bitstream/handle/10665/42716/9241546123.pdf\%0Awww.who.org

3. (World Health Orgnaisation) WHO, WHO (World Health Orgnaisation). Global prevalence of vitamin A deficiency in populations at risk WHO Global Database on Vitamin A Deficiency WHO Global Database on Vitamin A Deficiency. WHO Glob Database Vitam A Defic [Internet]. 2009;Geneva. Available from: http://www.who.int/vmnis

4. Stevens GA, Bennett JE, Hennocq Q, Lu Y, De-Regil LM, Rogers L, et al. Trends and mortality effects of vitamin A deficiency in children in 138 low-income and middle-income countries between 1991 and 2013: A pooled analysis of population-based surveys. Lancet Glob Heal. 2015;3(9):e528-36.

5. Imdad A, Mayo-Wilson E, Herzer K, Bhutta ZA. Vitamin A supplementation for preventing morbidity and mortality in children from six months to five years of age. Cochrane Database Syst Rev. 
2017;2017(3).

6. Jemberu Y, Zegeye M, Singh P, Abebe H. Consumption of Vitamin A Rich Foods and Associated Factors among Children Aged 6-23 Months in Kachabira. Int J Food Sci Nutr Eng. 2017;7(1):1-10.

7. Rakotonirainy $\mathrm{NH}$, Razafindratovo V, Remonja CR, Rasoloarijaona R, Piola P, Raharintsoa C, et al. Dietary diversity of 6- to 59-month-old children in rural areas of Moramanga and Morondava districts, Madagascar. PLoS One. 2018;13(7):1-14.

8. Dangura D, Gebremedhin S. Dietary diversity and associated factors among children 6-23 months of age in Gorche district, Southern Ethiopia: Cross-sectional study. BMC Pediatr [Internet]. 2017;17(1):17. Available from: http://dx.doi.org/10.1186/s12887-016-0764-x

9. Eshete T, Kumera G, Bazezew Y, Mihretie A, Marie T. Determinants of inadequate minimum dietary diversity among children aged 6-23months in Ethiopia: Secondary data analysis from Ethiopian Demographic and Health Survey 2016. Agric Food Secur [Internet]. 2018;7(1):1-8. Available from: https://doi.org/10.1186/s40066-018-0219-8

10. Aemro M, Mesele M, Birhanu Z, Atenafu A. Dietary diversity and meal frequency practices among infant and young children aged 6-23 months in Ethiopia: A secondary analysis of Ethiopian Demographic and Health Survey 2011. J Nutr Metab. 2013;2013.

11. WHO. Guideline: Vitamin A supplementation in infants and children 6-59 months of age. Who [Internet]. 2011;1-25. Available from: http://www.who.int

12. Central Statistical Agency Addis Ababa El. Ethiopia Demographic and Health Survey 2011 Central Statistical Agency [Internet]. 2012. Available from: http://www.measuredhs.com.

13. Fotheringham AS, Brunsdon C CM. Geographically Weighted Regression: The Analysis of Spatially Varying Relationships,. John Wiley Sons. 2003;35(3):272-5.

14. Krivoruchko K. Empirical bayesian kriging. Esri Redlands, CA, USA. 2012;

15. Kulldorff M. Theory and Methods A spatial scan statistic. Commun Stat. 1997;26(6)(February 2013):1481-96.

16. Fotheringham AS, Yang W, Kang W. Multiscale Geographically Weighted Regression (MGWR). Ann Am Assoc Geogr. 2017;107(6):1247-65.

17. Kenya National Bureau of Statistics Nairobi K. Demographic and Health Survey Kenya, 2014. 2014.

18. Ghana Statistical Service GHS and II. Ghana Health and Demographic Survey 2013. 2014.

19. International Institute for Population Sciences (IIPS) and ICF. National Family Health Survey (NFHS4), 2015-16: India. Mumbai: IIPS. For [Internet]. 2017. Available from: https://dhsprogram.com/pubs/pdf/FR339/FR339.pdf

20. (PhD) AK, General D, Kebede A, Modes T. Ethiopia National Food Consumption Survey. Ethiop Public Heal Inst [Internet]. 2013;3:54-67. Available from: http://www.ephi.gov.et

21. Harika R, Faber M, Samuel F, Mulugeta A, Kimiywe J, Eilander A. Are Low Intakes and Deficiencies in Iron, Vitamin A, Zinc, and lodine of Public Health Concern in Ethiopian, Kenyan, Nigerian, and South African Children and Adolescents? Food Nutr Bull. 2017;38(3):405-27. 
22. Baek $Y$, Chitekwe S. Sociodemographic factors associated with inadequate food group consumption and dietary diversity among infants and young children in Nepal. PLoS One. 2019;14(3):1-17.

23. Solomon D, Aderaw Z, Tegegne TK. Minimum dietary diversity and associated factors among children aged 6-23 months in Addis Ababa, Ethiopia. Int J Equity Health. 2017;16(1):1-9.

24. Edris MM, Atnafu NT, Abota TL. Determinants of Dietary Diversity Score among Children Age between 6-23 Months in Bench Maji Zone, Southwest Ethiopia. Pediatr Heal Res [Internet]. 2018;3(3):1-6. Available from: http://www.imedpub.com/articles/determinants-of-dietary-diversityscore-among-children-age-between-623-months-in-bench-maji-zone-southwest-ethiopia.php?aid = 23916

25. Tobler WR. A Computer Movie Simulating Urban Growth in the Detroit Region. Econ Geogr. 1970;46:234.

\section{Figures}

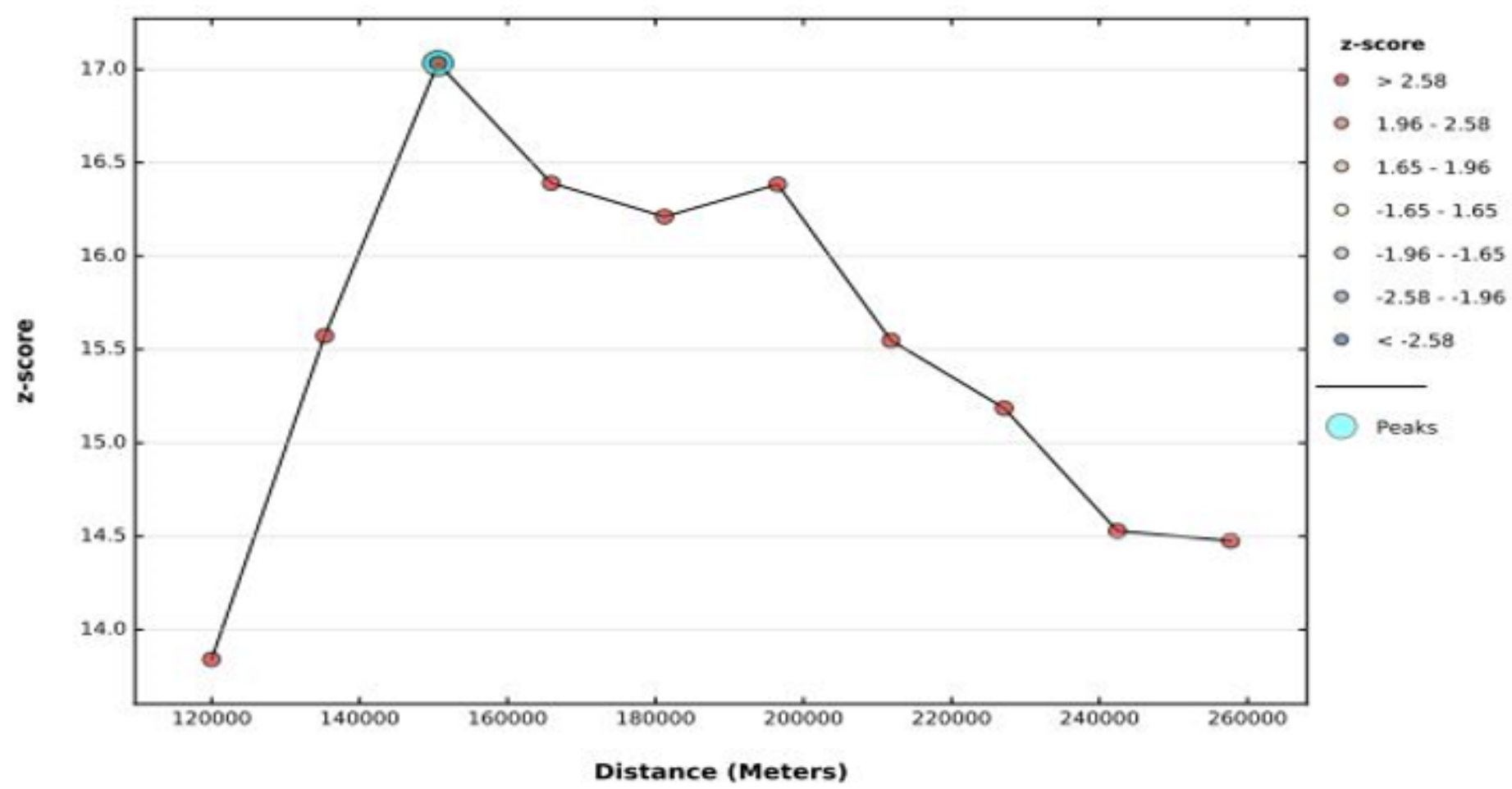

\section{Figure 1}

The spatial autocorrelation of poor consumption of foods rich in vitamin A among children age 6-23 months in 2016, EDHS, Ethiopia. 


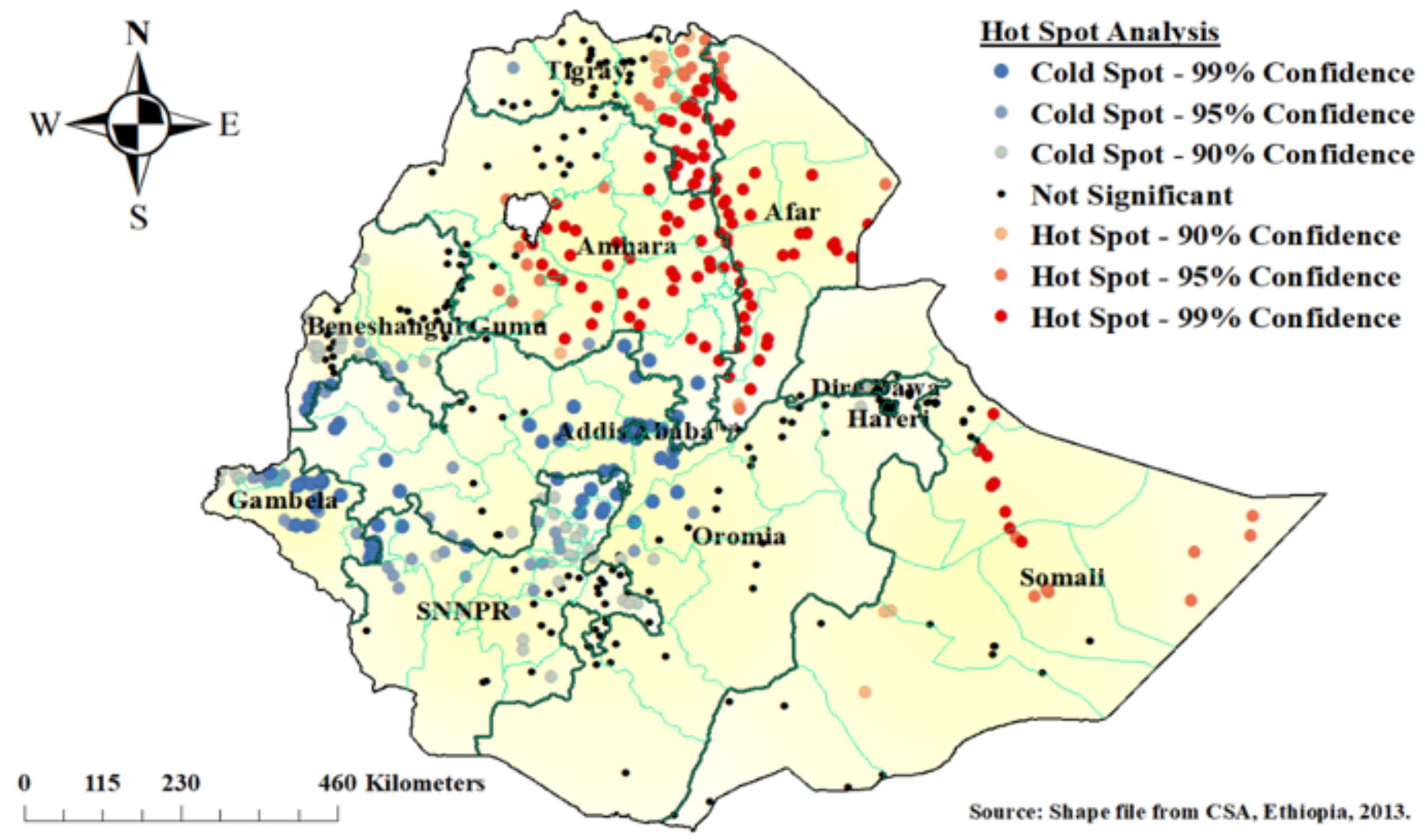

Figure 2

Hot spot analysis of poor consumption of foods rich in vitamin A among children age 6-23 months, 2016, EDHS.

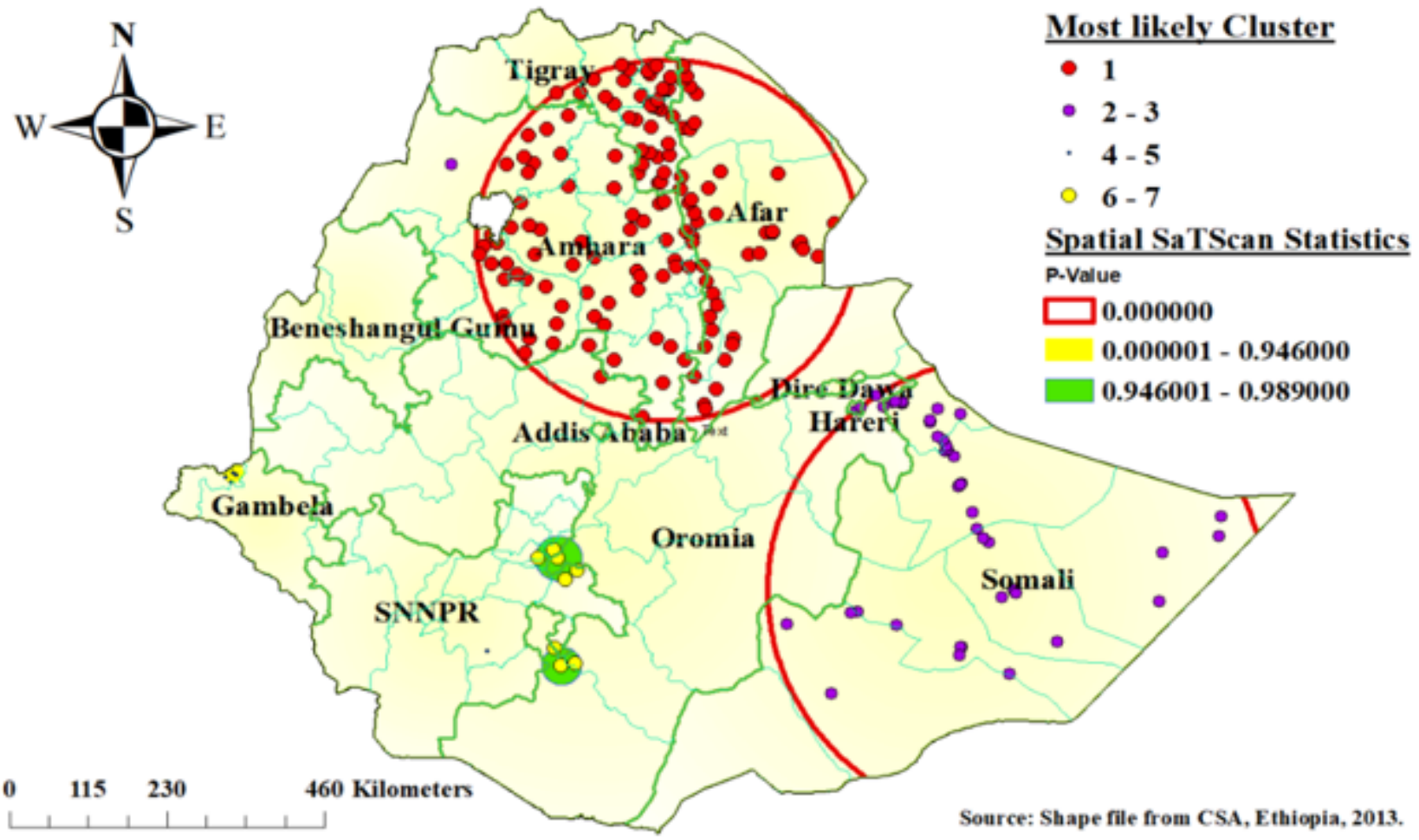




\section{Figure 3}

Most likely (primary) and secondary cluster for poor consumption of foods rich in vitamin A among children age 6-23 months, 2016, Ethiopia.

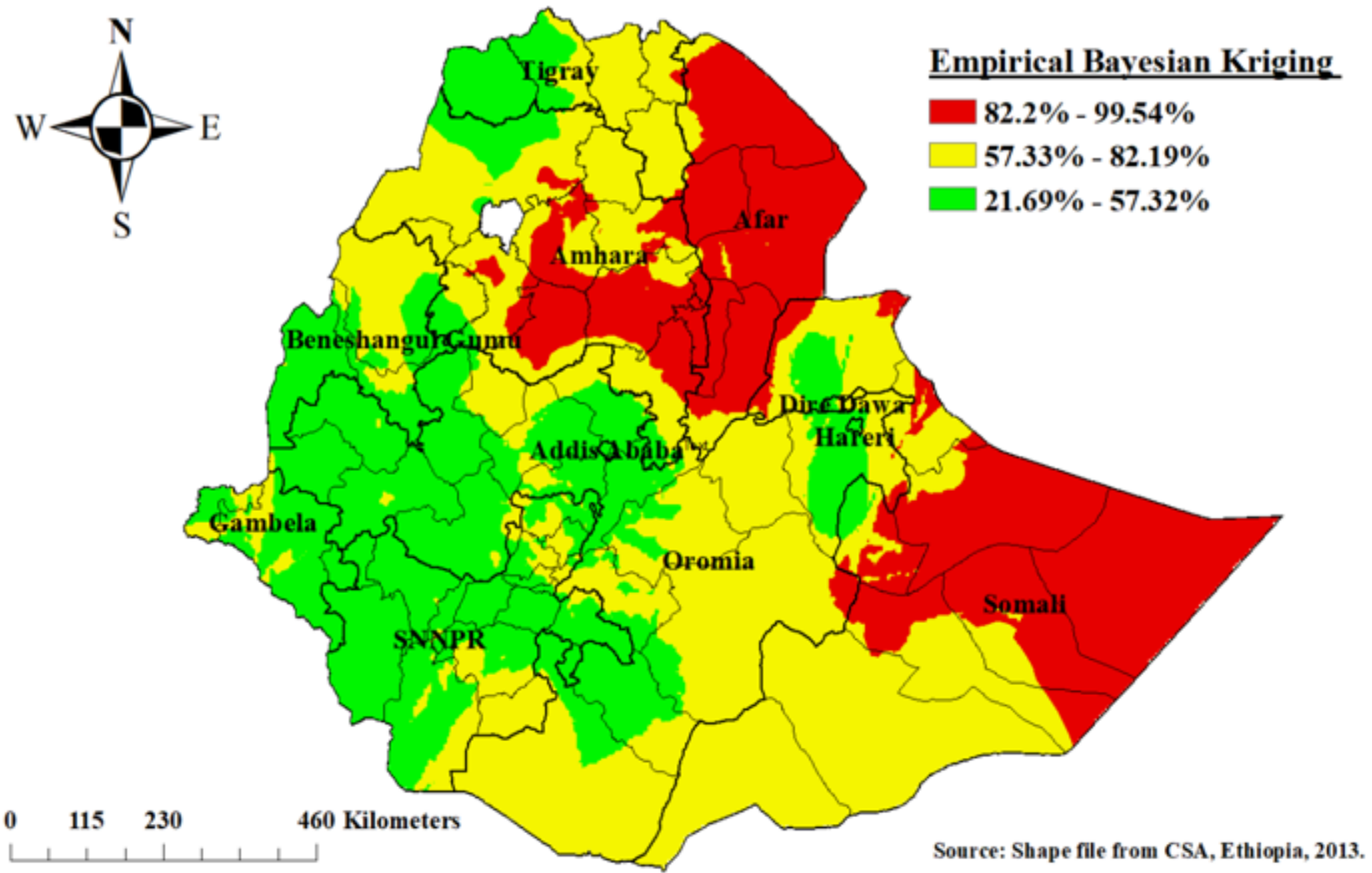

\section{Figure 4}

Empirical Bayesian Kriging interpolation of poor consumption of foods rich in vitamin A among children age 6-23 months, 2016, Ethiopia.

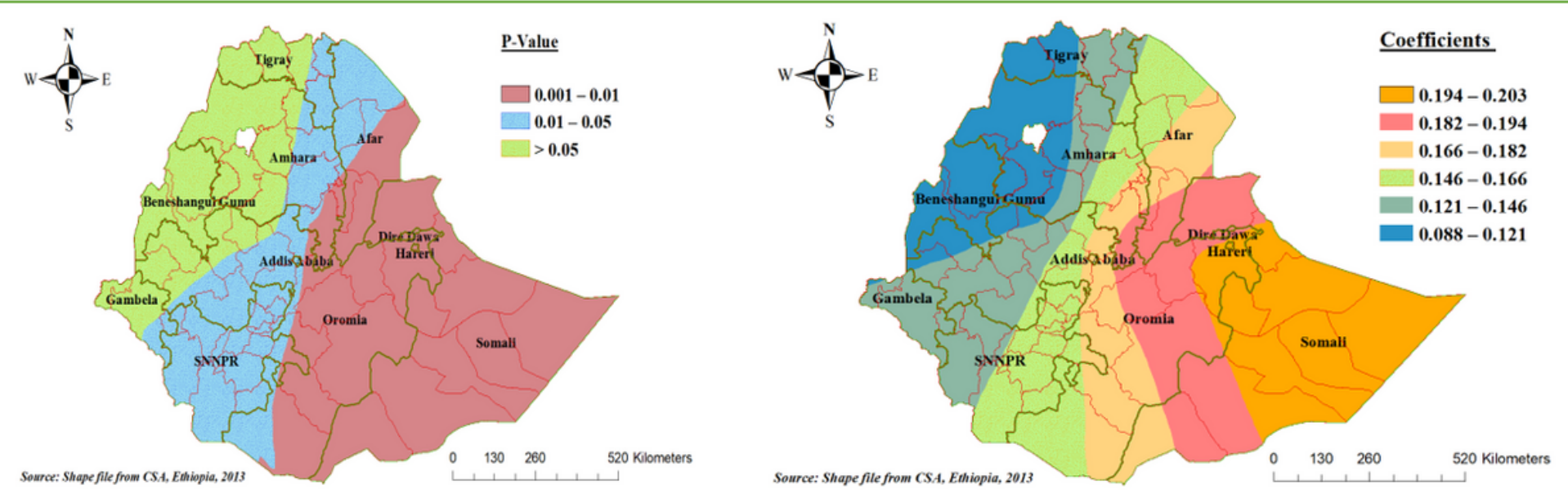




\section{Figure 5}

Geographically varying values of significance and coefficients per cluster for predictor variable poor wealth household of poor consumption in vitamin A rich foods in the final GWR model.

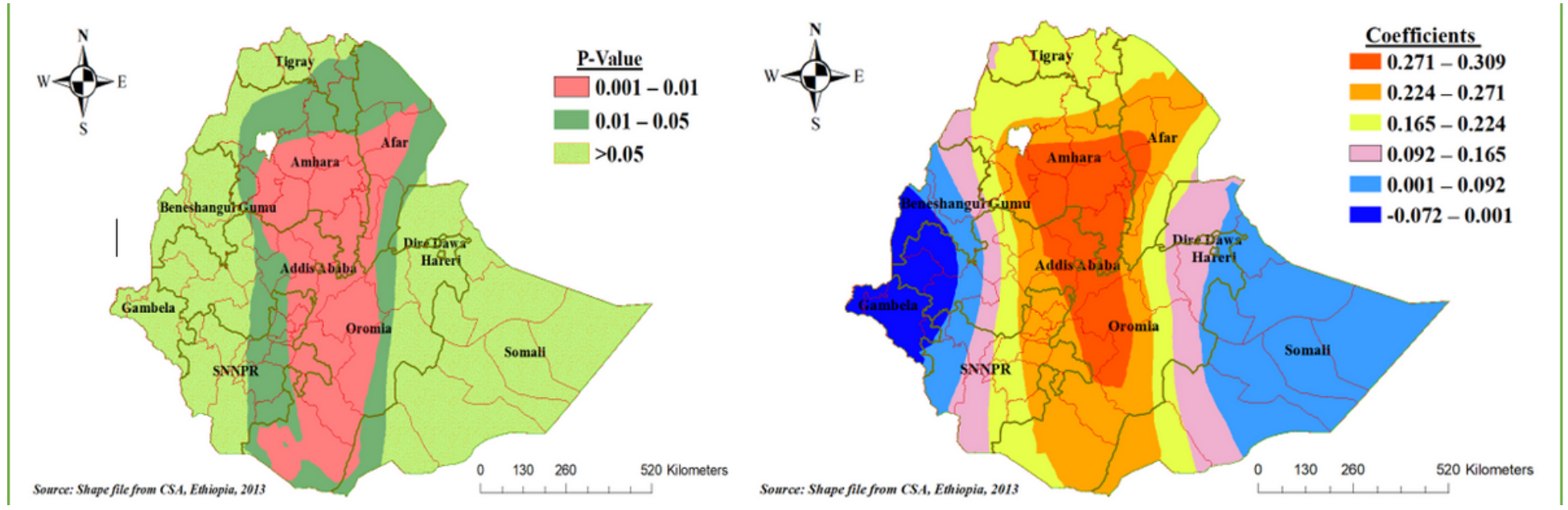

Figure 6

Geographically varying values of significance and coefficients per cluster for predictor variable rural residence of poor consumption in vitamin A rich foods in the final GWR model.

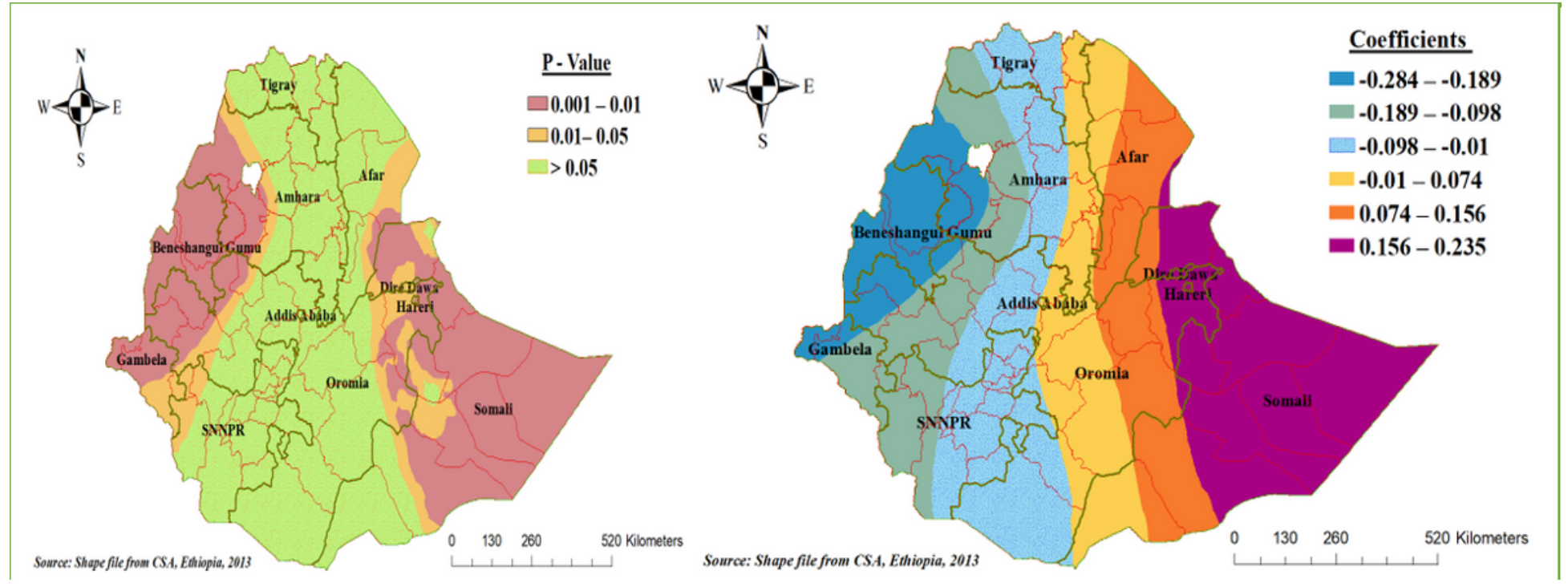

Figure 7

Geographically varying values of significance and coefficients per cluster for predictor variable living tropical area of poor consumption in vitamin A rich foods in the final GWR model.

\section{Supplementary Files}

This is a list of supplementary files associated with this preprint. Click to download. 
- Methodsformula.docx

Page 23/23 